\title{
Verstopfung bei Kindern
}

\section{In jedem Alter an Obstipation denken}

\author{
Rund 12\% der Kinder haben in ihren ersten Lebensjahren Symptome \\ einer Verstopfung. Wir sprachen mit Dr. Martin Claßen, Pädiatrische \\ Gastroenterologie, Klinik für Kinder- und Jugendmedizin Klinikum \\ Links d. Weser, über Ursachen, Therapien und die Rolle der Pflegenden.
}

? Wie oft "landen“ Kinder wegen Obstipation in der Notaufnahme, wie viele müssen stationär aufgenommen werden? Claßen: Dazu gibt es in Deutschland keine verlässlichen Daten. Man kann jedoch davon ausgehen, dass rund $12 \%$ der Kinder in ihren ersten Lebensjahren Symptome einer Verstopfung haben. Etwa 3\% der Vorstellungen beim Kinderarzt erfolgen aus diesem Grund. Andere Studien zeigen bei Kindern mit akutem Bauchschmerz eine Rate von 20-30\%, bei denen die Bauchschmerzen durch eine akute Verstopfung hervorgerufen und die Kinder unter Verdacht auf Appendizitis in der Klinik vorgestellt werden. Auch Kinder mit einer Stuhlinkontinenz leiden ursächlich häufig an einer Verstopfung.

? Welches Alter ist besonders betroffen? Claßen: Am häufigsten beginnt eine Verstopfung in der Altersgruppe, bei denen die Kontrolle über die Ausscheidungsorgane erlernt wird, also zwischen 1 und 4. Die Eltern bemerken die Probleme und suchen dann den Kinderarzt auf. Anders herum ist dies bei den Schulkindern; Schwierigkeiten beim Stuhlgang oder seltene Entleerungen fallen oft nicht auf. Die Vorstellung erfolgt dann eher mit anderen Hauptsymptomen. Daran denken muss man in allen Altersgruppen.

? Was ist häufigste Ursache der Obstipation - in Abhängigkeit vom Alter?

Claßen: Bei Neugeborenen und kleinen Säuglingen ist eine Fehlbildung des Enddarms, insbesondere der $M$. Hirschsprung in Betracht zu ziehen, bei formulaernährten Kindern auch eine Kuhmilchallergie. Ab dem Kleinkindalter spielt eine funktionelle Ursache die Hauptrolle. Am Anfang steht oft eine Irritation des Kindes am Anus oder im zeitlichen Zusammenhang mit der Defäkation.
Kinder finden die Entleerung unangenehm oder schmerzhaft, vermeiden dann die Stuhlentleerung. Der Stuhl wird hart, schmerzt bei der nächsten Entleerung. So kommt es zu einem Teufelskreis mit hartem Stuhl, Erweiterung des Enddarms, Stuhlrückhalt und fehlender Empfindung für den Drang. Jedes Kind mit einer chronischen Verstopfung muss noch auf eine Zöliakie untersucht werden.

? Was passiert nach der stationären Aufnahme?

Claßen: Bei jeder akuten Verstopfung muss man rasch für eine unproblematische, schmerzfreie Entleerung sorgen. Dies funktioniert am besten mit oralen Stuhlweichmachern; Macrogolpräparate sind das Mittel der Wahl. Bei jedem Kind mit einer chronischen, funktionellen Verstopfung erfolgt zunächst eine ausführliche Beratung über die Entstehung und die Behandlung. Hier kann man Informationsmaterial verwenden oder auch ein Video, das auf der Homepage der Kindergastroenterologen verfügbar ist (www.gpge.de - „The poo in You“).

Wenn größere Mengen Stuhl im Enddarm angesammelt sind, müssen diese beseitigt werden, bevor die Dauertherapie gelingen kann. Wir sehen das gut im Ultraschall. Dann kann man über drei Tage eine hohe Menge eines Stuhlweichmachers geben oder ein Klysma anwenden. Dabei darf man das Kind aber nicht weiter traumatisieren. Deshalb geben wir vorher Midazolam, um die Angst und die Erinnerung an das Klysma zu vermindern.

Die Dauertherapie besteht in einer Gabe von Macrogol, das von der Darmschleimhaut nicht aufgenommen wird und damit Wasser im Darm bindet. Die Dosis muss so hoch sein, dass der Stuhl cremig ist. Die Behandlung ist oft über Monate erforderlich, bis das Kind die Angst vor der Defäkation verliert.

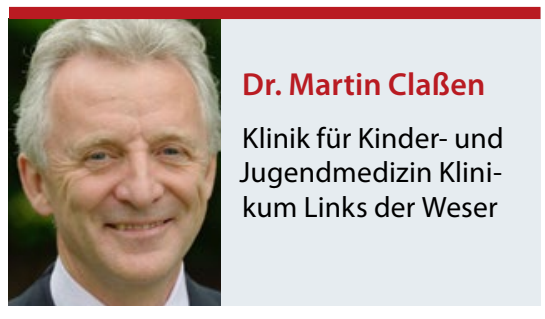

Thema sind auch immer Ernährung und Trinken. Wir raten zu löslichen Ballaststoffen in Obst (-säften, -schorlen), Reduktion der Milchmenge auf ein Glas pro Tag sowie von Schokolade etc. Die Beeinflussung reicht aber meist nicht aus, um eine Verstopfung in den Griff zu bekommen.

? Welche Aufgaben kommen der Pflege zu? Claßen: Bei der Betreuung von Kindern in der Tagesklinik gibt es bei uns immer eine separate Anamnese durch die Pflegenden und eine ausführliche Beratung über den Umgang mit dem Thema im Alltag. Auch die atraumatische Applikation von Klysmata wird von der Pflege umgesetzt. Die Toilettensitzungen werden mit den Eltern besprochen und ggf. mit den Kindern eingeübt. Ein Aspekt, auf den Pflegende besonders achten sollten, sind Veränderungen des Anus. Alle schmerzhaften Läsionen wie Entzündung, Einrisse etc. müssen erkannt und lokal behandelt werden.

Wenn die Behandlung der Verstopfung zu Hause nicht funktioniert, liegt das oft an völlig verfahrener Interaktion zwischen Eltern und Kind. In dem Fall haben wir sehr gute Erfahrung mit einer wenige Tage dauernden stationären Aufnahme mit Stuhltraining gemacht. In unserer Klinik gibt es drei Pflegekräfte mit einer Zusatzqualifikation als Urotherapeutin bzw. Kontinenztrainerin. Diese spielen in der Aufklärung, Schulung und Beratung der Kinder und der Eltern eine zentrale Rolle. Gut wäre es, wenn in allen Kinderkliniken spezialisierte Pflegekräfte dieses Thema vertreten würden.

Das Interview führte Ute Burtke. 